\title{
Erratum to: Analysis of 15 Cases of Auricular Keloids Following Conchal Cartilage Grafts in an Asian Population
}

Tae Hwan Park • Ji Hae Park • June-kyu Kim •

Sang Won Seo • Dong Kyun Rah • Choong Hyun Chang

Published online: 23 April 2013

(c) Springer Science+Business Media New York and International Society of Aesthetic Plastic Surgery 2013

Erratum to: Aesth Plast Surg 37(1):102-5

DOI 10.1007/s00266-012-9998-7

In the HTML version, the "Cosmetic" category should be replaced with "Original Article".

The online version of the original article can be found under doi:10.1007/s00266-012-9998-7.

T. H. Park - J. H. Park · J. Kim - S. W. Seo - C. H. Chang ( $\square)$ Department of Plastic and Reconstructive Surgery, Kangbuk Samsung Hospital, Sungkyunkwan University School of Medicine, 108 Pyung-Dong, Jongno-Gu, Seoul 110-746, Korea e-mail: eppeen@hanmail.net; hard-piano@hanmail.net

T. H. Park · D. K. Rah

Department of Plastic and Reconstructive Surgery, Institute for Human Tissue Restoration, Yonsei University, Seoul, Korea 